\title{
Recomendaciones para la atención fonoaudiológica en las áreas de audiología y otoneurología en contexto COVID-19: Una revisión narrativa
}

\author{
Anna María Morris a , Patricia Oyarzún ${ }^{\mathbf{b}}$, Sebastián Rivera ${ }^{\mathrm{c}, *}$ \\ a Escuela de Fonoaudiología, Universidad Santo Tomás, Sede Santiago, Chile \\ bscuela de Fonoaudiología, Universidad Santo Tomás, Sede Talca, Chile \\ ' Escuela de Fonoaudiología, Universidad Santo Tomás, Sede Viña del Mar, Chile
}

\section{RESUMEN}

La pandemia por el virus SARS-CoV-2 causante de la enfermedad COVID-19 se ha expandido rápidamente a nivel mundial. Las autoridades de salud pública han recomendado limitar las prestaciones de salud no esenciales, buscando el equilibrio entre el riesgo del retraso en el tratamiento y la potencial exposición al virus. En la mayoría de las prestaciones en las áreas de audiología y otoneurología no es posible cumplir con el distanciamiento físico recomendado y la duración de la atención puede superar los 45 minutos, factores que aumentan el riesgo de contagio para el profesional. El objetivo de la presente revisión es describir la evidencia actual sobre las recomendaciones de atención para las áreas de audiología y otoneurología en contexto COVID-19. Además de la búsqueda de artículos científicos en diversas bases de datos, se revisaron las recomendaciones emitidas por las principales asociaciones en el área. La información obtenida se organizó considerando cuatro aspectos clínicos relevantes: prestaciones, procedimientos, elementos de protección personal y limpieza de equipamiento e insumos. La evidencia científica y las sugerencias de las asociaciones están en constante actualización y algunos temas se han desarrollado escasamente. Sin embargo, las recomendaciones coinciden en priorizar la atención a distancia, extremar el uso de elementos de protección personal, implementar protocolos de higiene de manos y limpieza de equipos e insumos. También, se sugiere incorporar estrategias de comunicación alternativas al lenguaje oral con las personas con hipoacusia para facilitar la discriminación de la palabra.

\section{Recommendations for audiologist assistance in the areas of audiology and otoneurology in context COVID-19: A narrative review}

\section{ABSTRACT}

The COVID-19 pandemic caused by the SARS-CoV-2 virus has rapidly spread worldwide. Public health authorities have recommended limiting non-essential healthcare, in search of a balance between the risk of delaying treatment and the potential exposure to the virus. Most services in the audiology and otoneurology areas can easily exceed 45 minutes and it is not possible to keep the recommended physical distancing. Both factors increase the risk of professionals being infected. Therefore, the purpose of this review is to describe the current evidence about recommendations in audiology and otoneurology care in the context of COVID-19. In addition to the search of scientific articles in various databases, recommendations issued by the main associations in the area were consulted. The information was gathered considering four relevant clinical aspects: health care services, procedures, personal protective equipment and the cleaning of equipment and supplies. Scientific evidence and suggestions made by associations are being constantly updated, and therefore there is limited content on some topics. However, the recommendations agree on prioritizing remote health care, increasing the use of personal protective equipment, implementing protocols for hand hygiene and for the cleaning of equipment and supplies. The incorporation communication strategies other than oral language to interact with people with hearing loss to facilitate speech discrimination is also recommended.

*Autor/a correspondiente: Sebastián Rivera

Email:srivera@santotomas.cl

\section{Palabras clave:} Infecciones por Coronavirus; Pruebas de Función Vestibular; Audiología; Otoneurología

\section{Keywords:}

Coronavirus Infections; Vestibular Function Tests; Audiology; Neurotology 
La pandemia del COVID-19 causada por el virus SARS-CoV-2 se ha expandido rápidamente a nivel mundial desde los primeros casos notificados en la ciudad de Wuhan, China, a fines del año 2019. Reportes de la Organización Mundial de la Salud (OMS) indican que los casos confirmados ascienden a 12 millones de personas, incluidas alrededor de 500 mil muertes al mes de julio de 2020 (Organización Mundial de la Salud, 2020). Específicamente, al 27 de Julio de 2020, en Chile los contagios superan los 345 mil casos con más de 9000 fallecimientos (Ministerio de Salud, 2020), constituyendo un importante problema de salud pública a nivel nacional y mundial. Estas cifras posicionan al país en el quinto lugar de contagios totales y en el décimo lugar de los países con más fallecidos, por millón de habitantes (Organización Mundial de la Salud, 2020).

La ruta de transmisión predominante orienta principalmente al contacto directo persona a persona, o bien, por medio de gotas de saliva expulsadas al estornudar, toser o hablar (Rothan \& Byrareddy, 2020). Asimismo, se ha documentado la transmisión de contacto directo de las manos con superficies contaminadas, las que pueden posteriormente tocar la mucosa de los ojos, nariz y/o boca (Shi et al., 2020). Debido al carácter altamente contagioso, Adhikari et al. (2020), informaron que las principales medidas preventivas recomendadas para reducir la transmisión del virus son el lavado de manos, uso de mascarillas en espacios públicos, distanciamiento social, cuarentena, rastreo de datos, entre otros. Además, con el rápido crecimiento de la pandemia a nivel global y sin contar con vacuna o tratamiento definitivo, las autoridades de salud pública han recomendado limitar las prestaciones de salud no esenciales. Por consiguiente, la atención sanitaria debe determinar qué procedimientos son considerados "electivos", buscando el equilibrio entre el riesgo del retraso en tratamiento y la potencial exposición al COVID-19 (Pattisapu et al., 2020).

En la mayoría de las prestaciones en las áreas de audiología y otoneurología no se puede cumplir con el distanciamiento físico recomendado y la duración de la atención puede superar los 45 minutos, factores que aumentan el riesgo de contagio para el profesional. Por lo tanto, resulta de relevancia revisar la literatura internacional para conocer las distintas adecuaciones que se han realizado para las atenciones en el área en medio de esta emergencia sanitaria. De esta forma, el objetivo planteado para la presente revisión es describir la evidencia actual sobre las recomendaciones de atención para las áreas de audiología y otoneurología en el contexto COVID-19.

Para realiza la revisión, se utilizó una estrategia de búsqueda activa en las bases de datos como Pubmed, EBSCO y Scielo.
Asimismo, se revisaron páginas oficiales de las principales organizaciones a nivel nacional relacionadas con COVID-19 y de profesionales del área; recopilando información respecto a las prestaciones, procedimientos y cuidados sanitarios a considerar en la situación actual.

\section{PRESTACIONES EN LAS ÁREAS DE AUDIOLOGÍA Y OTONEUROLOGÍA}

Debido a los periodos críticos del desarrollo del lenguaje y al impacto a largo plazo de la deprivación auditiva, algunos servicios de audiología deberían ser considerado esenciales (Pattisapu et al., 2020). Gran dilema y variabilidad se ha generado respecto a la definición de los servicios esenciales versus servicios no esenciales. La Academia Americana de Audiología (AAA) declara que, a pesar de que los audiólogos no prestan servicios para mantener la vida según la definición de la crisis actual, no disminuye la importancia de su rol en la mantención de la calidad de vida de las personas (American Academy of Audiology, 2014). Por su parte, la Academia Americana de Otorrinolaringología afirma que la atención a personas sordas y con problemas auditivos debe considerarse urgente y esencial durante la pandemia (Moats \& Creel, 2020). Debido a que la audición es un sentido primordial para la comunicación, los pacientes dependen del diagnóstico y apoyo audiológico para mantenerse conectados y preservar su bienestar durante este periodo (HernándezGonzález et al., 2020).

En Hong Kong, desde febrero del año 2020, las consultas de pacientes ambulatorios se redujeron a un $10-20 \%$ en los servicios otorrinolaringológicos normales, para garantizar solo aquellas atenciones urgentes o semi urgentes como: cánceres de cabeza y cuello, emergencias de las vías respiratorias, abscesos de cabeza y cuello, otitis media aguda, mastoiditis aguda, sinusitis complicada, pérdida auditiva repentina, parálisis facial o trauma. Concurridas seis semanas se lograron reprogramar más del 95\% de las atenciones. Se aplicó telemedicina por la plataforma Zoom a los pacientes que no requerían una examinación física extensa, lo cual fue apropiado para el seguimiento de pacientes con síntomas nasales, dificultades auditivas, tinnitus, tratados por apnea del sueño, ronquera con causa benigna, mareos y afecciones plásticas faciales. Una evaluación preliminar sobre la satisfacción de los usuarios con esta tecnología fue prometedora, reflejando una excelente satisfacción general con un promedio de 9.1 de 10 , comunicación efectiva con un 9.2 de 10, experiencia de consulta satisfactoria con 9.2 de 10 y expectativa alcanzada con un 9.0 de 10 (Cho et al., 2020). 
Por su parte, el 9 de marzo del año 2020, el centro de control y prevención de enfermedades del Hospital de niños de Seattle (SCH) EEUU canceló los procedimientos no urgentes, minimizó el volumen clínico y utilizó la telemedicina cuando fuera posible. Varios hospitales de maternidad en el estado de Washington han interrumpido el examen auditivo neonatal y la evaluación de la audición ambulatoria durante la pandemia. Por esto, el SCH desarrolló un plan de divulgación para identificar a aquellos bebés que no fueron examinados al nacer y aquellos derivados para confirmación diagnóstica, cuya cita fue cancelada. Lo anterior debido a que se consideran esenciales estas atenciones, incluso durante la pandemia. Lo anterior basado en que 1 o 2 meses de retraso pueden tener implicaciones negativas para el lenguaje y desarrollo del paciente (Pattisapu et al., 2020).

A mediados de marzo del año 2020, en el Departamento de Otorrinolaringología-Cirugía Cabeza y Cuello del Centro Médico de la Universidad Rush en Illinois EEUU, todos los profesionales reprogramaron las citas y las convirtieron en atenciones de telemedicina, manteniendo de manera presencial solo los casos considerados críticos (West et al., 2020). En el mismo centro médico las prestaciones urgentes desde el punto de vista audiológico fueron limitadas a realizarse solo cuando el resultado del examen auditivo pudiera afectar de manera significativa la decisión médica. Un ejemplo de ellos son las hipoacusias sensorio-neurales súbitas (Batra et al., 2020).

La mayoría de los centros y asociaciones concuerdan en limitar la atención audiológica esencial a exámenes de detección y seguimiento de la audición a recién nacidos y servicios de audiología urgentes, como en pacientes que dependen de su dispositivo para escuchar y otras urgencias en servicios pre, peri y post operatorios (West et al., 2020). En este mismo sentido, no debería ofrecerse atención presencial a adultos sin síntomas auditivos durante la pandemia, en cambio, se les debería ofrecer una consulta remota digital (Association of Independent Hearing Healthcare Professionals et al., 2020).

En el SCH también se priorizó a aquellos pacientes sin acceso al habla o comunicación, incluyendo pacientes con pérdida auditiva sin audífonos o implantes cocleares (IC) funcionales y otras personas en quienes se ha sospechado cambios en su audición o que pueden aumentar el riesgo de pérdida auditiva. Además, se incluyen derivaciones consideradas urgentes por los servicios de hematología-oncología, craneofacial y otorrinolaringología para evaluar la pérdida auditiva resultante de la enfermedad o tratamiento médico. La pérdida auditiva unilateral no fue considerada una indicación urgente de audiometría, a menos que fuera de inicio súbito (Pattisapu et al., 2020).
Para la British Academy of Audiology (BAA) las prestaciones urgentes fueron: evaluación de pérdidas auditivas súbitas, evaluación de pacientes post meningitis y seguimiento de screening auditivo referidos bilateralmente en recién nacidos. Siempre que sea posible, recomiendan configurar los servicios de audiología para entregarse de manera remota, dejar citas presenciales o visitas domiciliarias como último recurso (British Academy of Audiology, 2020).

En una revisión narrativa realizada por Hernández-González et al. (2020) se hace referencia a las cirugías otológicas urgentes que deben atenderse entre 0 a 48 horas, considerando las complicaciones de otitis media y traumas del nervio facial y/o pabellón auricular. Las semi urgentes, con indicación de atención entre 48 horas a 1 mes serían: malignidad ósea temporal operable, implante coclear en pacientes con riesgo de osificación, descompresión del nervio facial por parálisis facial aguda, reparación de fistula de líquido cefalorraquídeo (LCR) de alto flujo, colesteatoma con parálisis del nervio facial o compromiso y tumores intracraneales con compresión del tronco encefálico. Desde el punto de vista audiológico se recomienda, en lo posible, realizar tele asesoría con video y evitar el contacto directo con el paciente, el cual debe realizarse únicamente en situaciones urgentes o prioritarias: otitis media complicada, sordera súbita idiopática y complicaciones postquirúrgicas. Recomiendan aplazar pruebas vestibulares y audiometrías no urgentes, excepto en hipoacusia súbita o inmunomediada. Las prestaciones esenciales o urgentes deben ser analizadas según el juicio profesional y cuando los beneficios superen los riesgos: mantenimiento y reparación de ayudas auditivas, reemplazo de ayuda auditiva extraviada, mantenimiento y reparación de dispositivo de implante de conducción ósea, cuidados del sitio operatorio del implante de conducción ósea, tamizaje auditivo neonatal y seguimiento, encendido o programación de IC, necesidades audiológicas específicas individualizadas, evaluación post meningitis y solicitudes individuales por otorrinolaringología-otología.

Las prestaciones que pueden ser realizadas de manera remota incluyen: screening auditivo en adultos, reemplazo o actualización de audífonos para usuarios experimentados, calibración de audífonos, consejos de rehabilitación y seguimiento auditivo, evaluación del tinnitus, identificación de síntomas o signos para determinar la urgencia de atención, rehabilitación y seguimiento vestibular (Association of Independent Hearing Healthcare Professionals et al., 2020; Pattisapu et al., 2020). Según la British Academy of Audiology (2020) las prestaciones que pueden ser reemplazadas por una consulta por video o telefónica son: consejería y apoyo respecto 
al tinnitus, rehabilitación vestibular y seguimiento de audífonos. Pacientes usuarios de dispositivos de ayuda auditiva que tengan sospecha de rotura, falla o disfunción de alguno de los componentes del dispositivo o adaptación acústica (moldes, codos, tubos, domos, micro moldes, etc.). Se recomienda coordinar la entrega-recepción del dispositivo completo a fin de intentar resolver la necesidad sin la atención presencial del paciente.

Por su parte, la Academia Brasileña de Audiología (ABA) prioriza la atención remota en relación a la condición del paciente, por lo que se debe realizar en: pacientes con habilidades tecnológicas necesarias para este tipo de atención, pacientes estables con apoyo familiar y que presentan buena evolución en el tratamiento y pacientes considerados grupo de riesgo para el COVID-19 (Academia Brasileira de Audiología, 2020). En el caso de la Asociación Argentina de Audiología (ASARA), desaconseja atenciones a domicilio por la imposibilidad de controlar las variables de higiene y bioseguridad del entorno de atención en el domicilio o residencias (Asociación Argentina de Audiología, 2020).

La revisión de la literatura realizada por Hernández-González et al. (2020) sintetizó diversos ámbitos referidos a la atención de pacientes. De acuerdo a la revisión, los servicios remotos podrían abordar la evaluación y consejería del tinnitus, ajuste de ayudas técnicas, rehabilitación auditiva, seguimiento y consejería con respecto al manejo no presencial del cerumen. Las atenciones remotas en el área vestibular podrían utilizarse en pacientes con Vértigo Posicional Paroxístico Benigno (VPPB) a los que se les podría aplicar un screening remoto sin la necesidad de equipamiento. Si es necesario se remite a terapia vestibular o si son autónomos sin riesgo de caídas se entregan ejercicios de habituación. El mismo criterio se puede utilizar con los pacientes con diagnóstico de Enfermedad de Ménière sin crisis significativas o frecuentes. Para personas mayores con múltiples comorbilidades reagendar o establecer tiempos específicos para ellos. Respecto a las visitas domiciliarias de rehabilitación, se sugiere comenzar con una visita virtual para generar la priorización, si también se necesita un plan de rehabilitación vestibular éste puede ser entregado de manera remota (Rizk et al., 2020). En definitiva, siempre que sea posible, se recomienda configurar los servicios de audiología para entregarse de manera remota, las citas presenciales y domiciliarias se deben evitar al máximo (British Academy of Audiology, 2020).

Con respecto a la evaluación auditiva neonatal, la ABA recomienda que en la totalidad de los casos de diagnóstico temprano de la pérdida auditiva se realice atención presencial, con todos los cuidados necesarios (Academia Brasileira de Audiología, 2020). La evaluación auditiva de recién nacidos se puede realizar en el caso que la madre no presente síntomas de COVID-19. Cuando el procedimiento es realizado, debe ejecutarse por un personal que no trabaje directamente con pacientes con COVID-19 (Krajewska et al., 2020).

En el SCH se priorizó la programación de potenciales evocados auditivos de tronco cerebral (PEATC) para la confirmación diagnóstica de bebés que hayan referido en ambos oídos en el screening auditivo. En cuanto a los dispositivos auditivos, se recomienda seguir programando la adaptación para pacientes con hipoacusia bilateral, para asegurar el acceso a la comunicación. La adaptación se realiza en base al PEATC que tenga el paciente a los 6 meses de edad, por lo que aquellos que se acercan a esas fechas deben ser priorizados como urgentes (Pattisapu et al., 2020). Sin embargo, el tiempo que transcurre para realizar la confirmación diagnóstica de un paciente que refiere en el screening auditivo debe adecuarse a cada institución. La American Speech-Language-Hearing Association (ASHA) recomienda esperar hasta que la condición sanitaria sea segura. Además, se propone priorizar aquellos que presenten tiempos que estén al límite de lo aconsejable, considerando la confirmación diagnóstica a los 3 meses e ingresar a servicios de intervención temprana a los 6 meses de edad (American Speech-LanguageHearing Association, 2020a). Finalmente, los bebés que refirieron el screening auditivo, durante los periodos de restricción, deben recibir la máxima prioridad para los servicios presenciales tan pronto como sea posible. Respecto a acciones futuras, se sugiere una rúbrica para evaluar cada tipo de servicio individual, apoyándose de que la pérdida auditiva pediátrica es una emergencia neurológica. Debe actualizarse, estandarizarse y ser ampliada para apoyar en futuras situaciones donde la presencialidad no sea factible, considerando que el screening auditivo y las pruebas diagnósticas de seguimiento no están disponibles o no son factibles en un modelo de tele salud.

Es importante destacar que aún no se puede medir el real impacto de las recientes interrupciones de los servicios de detección y seguimiento de los programas de screening auditivo (Moats \& Creel, 2020).

\section{PROCEDIMIENTOS EN LAS ÁREAS DE AUDIOLOGÍA Y OTONEUROLOGÍA}

La evidencia actual carece de recomendaciones específicas sobre adecuaciones en los procedimientos de las áreas de audiología y otoneurología. 
Goldin et al. (2020) estudiaron las diferencias en la comprensión del habla a nivel acústico en función de la variedad de mascarillas existentes en el mercado. Así hubo un grupo que usó mascarilla simple (quirúrgica), otro grupo con N95 común y un tercer grupo que utilizó mascarilla con N95 con filtro. Estos grupos se compararon con un grupo control que no utilizó mascarilla. Los resultados mostraron que, en general, las mascarillas actúan como un filtro de pasa bajo atenuando las altas frecuencias de 2000 a $7000 \mathrm{~Hz}$. Por tanto, ocurre una disminución de 3-4 dB SPL al utilizar una mascarilla simple y de $12 \mathrm{~dB}$ SPL aproximadamente para una N95. En conclusión, la degradación de la calidad del habla, sumado con la ausencia de pistas visuales y el ruido de fondo de las salas, afectan negativamente la inteligibilidad de la palabra. En este mismo sentido, West et al. (2020) se refieren a la importancia de proporcionar información visual cuando se utiliza mascarilla, proponiendo como una alternativa viable el uso de mascarilla transparente para generar el apoyo en la lectura labio facial (LLF).

Atcherson et al. (2017) también se refieren a la importancia de las ayudas visuales para las personas con hipoacusia y el desmedro que genera para la comunicación el uso de mascarillas convencionales. Estudiaron a un grupo de personas con y sin hipoacusia, a las cuales se les solicitó repetir una lista de oraciones en diferentes condiciones, con/sin mascarilla convencional y con mascarilla transparente frente a situaciones de ausencia o presencia de ruido de fondo. Concluyeron que las mascarillas transparentes no afectan el rendimiento de la percepción de la palabra en ruido debido a las ayudas visuales que proporciona la LLF.

El Center for disease Control and Prevention (2020) declara que las personas con problemas de audición, o aquellas que cuidan e interactúan con una persona con discapacidad auditiva, pueden disminuir el uso de mascarillas convencionales debido a su dependencia con la LLF para comunicarse. En esta situación se debe proponer el uso de escudo facial que permita visualizar las expresiones faciales y movimientos de labios. Si no se cuenta con este implemento, se puede escribir el mensaje y/o disminuir el ruido de fondo para favorecer la comunicación.

La American Speech-Language-Hearing Association (2020b) insta a reconocer y promover la importancia de poner a disposición de las personas métodos de comunicación flexibles. A modo de ejemplo, se sugiere el uso de pantallas de computadora, block de notas, pizarras, aplicaciones de voz, de barreras de plexiglás y otros recursos que puedan ayudar a que la comunicación sea más efectiva. Asimismo, resultaría útil proporcionar a los pacientes las instrucciones por escrito para la realización de los exámenes (Asociación Argentina de Audiología, 2020).

El distanciamiento físico también crea desafíos para la comunicación con las personas con dificultades auditivas. En este sentido, el volumen de la voz disminuye con la distancia y resulta complejo el uso de las señales visuales como los movimientos de los labios y las expresiones faciales. La American SpeechLanguage-Hearing Association (2020b) recomienda favorecer la comunicación. Para ello se debe asegurar tener la atención de la persona, hablar un poco más fuerte y más despacio, acompañar la comunicación con lenguaje corporal y monitorear si la persona comprendió el mensaje.

Finalmente, la Massachusetts Commission for the Deaf and Hard of Hearing $(\mathrm{MCDHH})$ proporciona una guía que permite que profesionales y personas con hipoacusia o sordas puedan comunicarse mejor en situaciones de hospitalización por coronavirus. La guía incorpora imágenes y pictogramas, denominados Covid-19 Card que permiten a los profesionales consultar por sintomatología y conocer la modalidad de comunicación que prefiere la persona (Massachusetts Commission for the Deaf and Hard of Hearing, 2020).

\section{ELEMENTOS DE PROTECCIÓN PERSONAL Y LIMPIEZA DE EQUIPAMIENTO E INSUMOS}

Las atenciones de las áreas de audiología y otoneurología requieren en general un contacto directo con el paciente. Junto con ello, se suma el uso de implementos que exigen el contacto de múltiples usuarios. Por lo anterior, se torna imprescindible la necesidad de tomar medidas que minimicen los riesgos de transmisión de infecciones. Esto cobra mayor relevancia cuando se considera que quienes requieren de servicios de diagnóstico y rehabilitación en el área suelen tener factores que influyen negativamente en el sistema inmune, tales como edad avanzada, enfermedad de base y uso de fármacos (American Academy of Audiology, 2014). En el caso de que los procedimientos deban efectuarse, es necesario respetar los 2 metros de distancia, minimizar al máximo el tiempo de la consulta y realizar únicamente lo necesario (Hernández-González et al., 2020).

Cualquier programa de control de infecciones incluye la higiene de manos como una parte central y eficaz para evitar contagios. Se recomienda seguir el protocolo de lavado o desinfección de manos indicado por la Organización Mundial de la Salud (2009). Se indica emplear un preparado de base alcohólica o agua y jabón, siendo este proceso más eficaz cuando la piel de las manos se 
encuentra libre de cortes, las uñas son cortas, naturales, sin esmalte; las manos y los antebrazos están libres de joyas y al descubierto. Se propone una visión unificada para los profesionales de salud con el fin de minimizar la variación entre individuos. Para ello, se establecen cinco momentos de la higiene de manos: antes de contactarse con el paciente, antes de realizar una tarea limpia, luego de tener contacto con fluidos corporales, después del contacto con el paciente y posterior al contacto con el entorno del usuario.

En cuanto a la vestimenta empleada para realizar las atenciones, el National Hearing Conservation Program (2020) recomienda el uso de uniforme clínico completo, el cual debe ser de uso exclusivo dentro del establecimiento de salud, además de ser lavado diariamente. Si bien no se ha determinado los tejidos más efectivos contra la contaminación por virus, es conveniente usar vestimenta que tenga una composición mayor de algodón que poliéster o nylon. Al respecto, la British Academy of Audiology (2020) recomienda no usar ropa de mangas bajo el codo para facilitar la limpieza de las manos.

\section{ELEMENTOS DE PROTECCIÓN PERSONAL}

Se han propuesto una serie de sugerencias respecto a la protección personal. La American Academy of Audiology (2014) recomienda usar guantes durante los procedimientos de manejo del cerumen y de irrigación, cuando el paciente tenga un oído drenante, presencia de sangre, llagas o lesiones evidentes en el pabellón auricular o cuando el historial médico indique presencia de patologías infecciosas. También se sugiere usar guantes al desinfectar un área contaminada. Después de su uso, los guantes se desechan y se debe realizar un lavado de manos de manera inmediata. Los anteojos de seguridad están indicados cuando el audiólogo se encuentra expuesto a salpicaduras de material potencialmente infeccioso, por lo cual la extracción del cerumen mediante irrigación puede requerir su uso.

La British Academy of Audiology (2020) y la American SpeechLanguage-Hearing Association (2020a) han enfatizado la protección facial. Así, la British Academy of Audiology (2020) indica que la protección ocular es reutilizable, pero debe limpiarse entre cada uso y nunca debe compartirse entre los profesionales. Por su parte, la American Speech-Language-Hearing Association (2020a) recomienda que los usuarios usen escudo facial para la toma de impresiones del molde del oído o procedimientos que puedan provocar un reflejo de tos del paciente.
El National Hearing Conservation Program (2020) ha planteado que se deben preferir las mascarillas FFR N95, ya que ofrecen protección respiratoria contra la inhalación de gotas pequeñas que contienen SARS-CoV-2. De no estar disponibles, es necesario usar una mascarilla desechable de tipo quirúrgica con bucle diseñado para cubrir la boca y la nariz. Estas últimas no proporcionan protección respiratoria, pero entregan una barrera física para bloquear grandes gotas de fluidos corporales. Como se mencionó previamente las mascarillas reducen la eficacia de la comunicación oral al amortiguar el sonido y eliminar las señales visuales que facilitan la comprensión del habla. Lo anterior produce que la comunicación sea más difícil para todos, pero especialmente para las personas con trastornos auditivos. La American Speech-Language-Hearing Association (2020b) plantea que una máscara facial transparente puede ser útil para algunas personas. Sin embargo, en muchos casos las claves visuales no compensarán las dificultades creadas por el volumen reducido y la distorsión del mensaje.

Respecto a los procedimientos vestibulares, la Association of Independent Hearing Healthcare Professionals et al. (2020) establecen recomendaciones orientadas al uso de elementos de protección personal. En particular, sus indicaciones son para la evaluación y tratamiento del VPPB y la realización de la prueba calórica, pues se considera que estas pueden provocar náuseas, tos y/o vómitos, lo que aumentaría el riesgo de contagio. Asimismo, sugieren que el examinador debería contemplar uso de lentes de seguridad, guantes, delantal y mascarilla quirúrgica resistente a fluidos tipo IIR (protección media contra salpicaduras) durante toda la prueba. Esto dado la proximidad evaluador-paciente y el tiempo de ejecución. Por otra parte, para la ejecución del videoHead Impulse Test (v-HIT), videonistagmografía (VNG), silla rotatoria, potenciales evocados vestibulares miogénicos cervicales/oculares (c/oVEMPs) y posturografía se recomienda el uso guantes, delantal y mascarilla quirúrgica resistente a fluidos tipo IIR. No obstante, el uso de lentes de seguridad es opcional, y se sugiere principalmente cuando el paciente se niega a usar mascarilla.

\section{LIMPIEZA DE EQUIPAMIENTO E INSUMOS}

Los establecimientos de atención audiológica deben usar un desinfectante de grado hospitalario el cual puede estar en forma de toallitas, en aerosol o en sustancias de remojo en una bandeja o una máquina ultrasónica. Es importante emplear productos desinfectantes que no desnaturalicen químicamente el plástico, silicona, caucho y acrílico. El Environmental Protection Agency 
(EPA) de EEUU ha indicado el alcohol isopropílico como un desinfectante adecuado para ser usado contra el SARS-CoV-2 (Envoiromental Protection Agency, 2020).

En el contexto de atención audiológica es aceptable la desinfección en objetos que no están en contacto con sustancias potencialmente infecciosas, es decir, cerumen, drenaje de oído o sangre. La British Academy of Audiology (2020) recomienda usar desinfectantes a base de alcohol en concentraciones de 70-80\%. Por otra parte, la American Academy of Audiology (2014) señala que las curetas utilizadas en la extracción de cerumen y los espéculos de los otoscopios deben desecharse o esterilizarse, cuando estén contaminados con cerumen, drenaje del oído o sangre. Dado lo antes mencionado, aquellos insumos que tiene potencial de ser contaminantes cruzados se deben priorizar en formato desechable (espéculos de otoscopio, olivas de impedanciómetros y emisiones otoacústicas puntas de jeringas de impresión de moldes, auriculares de inserción y cubiertas de auriculares). Por su parte, la British Academy of Audiology (2020) sugiere que la remoción de cerumen no debería realizarse por los audiólogos en estos momentos. Por su parte, la British Academy of Audiology (2020) sugiere que los audiólogos no deben realizar remoción de cerumen en estos momentos.

Para la realización de la examinación otoscópica se sugiere utilizar un videotoscopio, ya que permite mantener una mayor distancia con el paciente. Se debe enfatizar en que todos los componentes y accesorios de los equipos de evaluación audiológica (impedanciómetro, potencial evocado auditivo, otoemisor, analizador de audífonos e interfaces de calibración) deben ser desinfectados posterior a la atención de cada paciente.

En caso de que no interfiera con la práctica clínica, es recomendable mantener la puerta abierta de la sala de atención, para posibilitar una mejor ventilación. Se debe reducir el número de pacientes, lo cual permite asegurar la desinfección y limpieza entre cada procedimiento realizado (Rizk et al., 2020). Las áreas manipuladas con frecuencia (manillas de puertas, reposabrazos, sillas, superficies de escritorio, teclados, teléfonos, ratones, portapapeles, interruptores de luz, vidrio de la cabina) y otras áreas comunitarias deben desinfectarse de manera rutinaria. El producto que se emplee debe dejarse secar y evaporar por diez minutos. Usualmente los pisos en las clínicas de audiología son alfombrados, por la necesidad de absorción del sonido y las directrices sobre la limpieza de la tela es limitado ya que rara vez se usan en otros entornos de atención médica. Si bien lo más recomendado es buscar orientación de los departamentos locales de control de infecciones, se sugiere usar desinfectante en aerosol, considerando luego un tiempo de ventilación (British Academy of Audiology, 2020).

La Asociación Argentina de Audiología (2020) enfatiza en que las cabinas audiológicas son espacios con un alto riesgo de acumulación de carga vírica y de difícil desinfección. La Sociedad Española de Otorrinolaringología y Cirugía de Cabeza y Cuello (SEORL) sugiere priorizar el uso de auriculares de inserción desechables, en caso de no contar con ellos es necesario usar protectores desechables sobre los auriculares de material acústico y permeable (Lavilla et al., 2020). Además, se recomienda desinfección de los componentes que se tocan con frecuencia después de cada sesión de evaluación, como son: cables, auriculares y pastilla ósea (American Academy of Audiology, 2014). El micrófono del paciente tiene que cubrirse con una bolsa plástica, la cual se cambia luego de cada atención. Este procedimiento debe ir seguido por una ventilación cuya duración dependerá del tamaño del espacio. Sin embargo, se debe tener de referencia el tiempo utilizado en la ventilación de una habitación de hospital, que es de 30 minutos (Lavilla et al., 2020). El auricular cintillo que utiliza el audiólogo para el retorno auditivo no debe ser compartido con otros examinadores. En el caso de evaluar a niños es necesario cuidar que los materiales usados para su condicionamiento se puedan desinfectar posteriormente.

Respecto a los cuidados del audífono y procesadores de implantes cocleares, cuentan con indicaciones de mantenimiento diario, las cuales deben mantenerse en el contexto de pandemia. Sin embargo, al considerar que pueden estar expuestos a ser contaminados por el virus la mayoría de los fabricantes coinciden en que se pueden tomar algunas medidas adicionales en relación con su limpieza. En este sentido, se indica que al tener la sospecha de que el dispositivo está contaminado, se sugiere realizar limpieza con toallas de alcohol isopropílico, seguido por un secado cuidadoso con tela. Asimismo, se orienta a los usuarios a no realizar limpieza en los micrófonos ni porta pilas. Lo anterior debido a que no existen estudios acerca del efecto que puede tener el uso a largo plazo de desinfectantes sobre el material biocompatible con el cual están fabricados los dispositivos (British Association of Teachers of the Deaf \& UK Assistive Listening Technology Working Group, 2020).

\section{COMENTARIOS DE LOS AUTORES}

La evidencia científica y las sugerencias por parte de las asociaciones de profesionales están en constante actualización, por lo que algunos temas aún están escasamente abordados. La esencialidad y urgencia son los principales temas de discusión en 
las áreas de audiología y otoneurología. A pesar de que las atenciones que se determinen como indispensables no implican un riesgo vital, es de común acuerdo que existe un gran impacto en la calidad de vida y comunicación de los pacientes.

La mayoría de las atenciones se han postergado por la contingencia y sus verdaderas consecuencias a largo plazo aún siguen siendo desconocidas. La evaluación auditiva en hipoacusias súbitas, el screening auditivo neonatal y la confirmación de hipoacusia en los casos bilaterales, son las principales prestaciones consideradas urgentes y que deberían mantenerse en situación de contingencia sanitaria. En la ejecución del resto de las atenciones se debe priorizar la modalidad de telepráctica y/o consejería a distancia.

Es destacable el cuidado tanto del audiólogo como del paciente. Ello implica utilizar de manera estricta los elementos de protección personal y realizar una correcta y permanente limpieza de manos, insumos, equipos y salas de atención. Finalmente, no existen sugerencias respecto a adecuaciones en los procedimientos audiológicos y otoneurológicos. Sin embargo, se enfatiza que, en el contexto de la pandemia, el uso de mascarillas y distanciamiento físico implica la necesidad de incorporar adaptaciones en la comunicación con las personas con hipoacusia.

\section{REFERENCIAS}

Academia Brasileira de Audiología. (2020). Recomendações para o retorno da prática em Audiologia. https://www.audiologiabrasil.org.br/portal2018/pdf/abant-recomendacoes-para-o-retorno-da-pratica-em-audiologia.pdf

Adhikari, S. P., Meng, S., Wu, Y.-J., Mao, Y.-P., Ye, R.-X., Wang, Q.-Z., Sun, C., Sylvia, S., Rozelle, S., Raat, H., \& Zhou, H. (2020). Epidemiology, causes, clinical manifestation and diagnosis, prevention and control of coronavirus disease (COVID-19) during the early outbreak period: A scoping review. Infectious Diseases of Poverty, 9(1), 29. https://doi.org/10.1186/s40249-020-00646-x

American Academy of Audiology. (2014, junio 12). Infection Control in Audiological Practice. Audiology. https://www.audiology.org/publications/guidelines-and-standards/infectioncontrol-audiological-practice

American Speech-Language-Hearing Association. (2020). Audiology Service Delivery Considerations in Health Care During COVID-19. American SpeechLanguage-Hearing Association. https://www.asha.org/Practice/AudiologistsResuming-Services-During-COVID-19/

American Speech-Language-Hearing Association. (2020). Communicating Effectively While Wearing Masks and Physical Distancing. American SpeechLanguage-Hearing Association. https://www.asha.org/public/CommunicatingEffectively-While-Wearing-Masks-and-Physical-Distancing/
Asociación Argentina de Audiología. (2020). Guía de recomendaciones para la prestación de servicios audiológicos bajo contingencias ligadas a la pandemia COVID-19. http://www.asara.org.ar/article.php?q=0000000096

Association of Independent Hearing Healthcare Professionals, British Academy of Audiology, British Society of Audiology, \& British Society of Hearing Aid Audiologists. (2020). Audiology and otology guidance during Covid-19. https://www.baaudiology.org/app/uploads/2020/06/Joint-Guidance-30thJune.pdf

Atcherson, S., Mendel, L., Baltimore, W., Patro, C., Lee, S., Pousson, M., \& Spann, M. (2017). The Effect of Conventional and Transparent Surgical Masks on Speech Understanding in Individuals with and without Hearing Loss. Journal of the American Academy of Audiology, 28(1), 58-67. https://doi.org/10.3766/jaaa.15151

Batra, P. S., LoSavio, P. S., Michaelides, E., Revenaugh, P. C., Tajudeen, B. A., Al-khudari, S., Husain, I., Papagiannopoulos, P., Smith, R., Stenson, K. M., \& Wiet, R. M. (2020). Management of the Clinical and Academic Mission in an Urban Otolaryngology Department During the COVID-19 Global Crisis. Otolaryngology-Head and Neck Surgery, 163(1), 162-169. https://doi.org/10.1177/0194599820929613

British Academy of Audiology. (2020). British Academy of Audiology Response to Covid-19: NHS England Essential Activity for Audiology. https:/www.baaudiology.org/app/uploads/2020/04/BAA-response-to-covid-19NHS-England-Essential-Activity-for-Audiology-update-31st-March.pdf

British Association of Teachers of the Deaf, \& UK Assistive Listening Technology Working Group. (2020). Cleaning Hearing Devices and Radio Aids Supporting Health and Safety During COVID-19. https://www.batod.org.uk/wpcontent/uploads/2020/03/Cleaning-hearing-devices-and-radio-aids-supportinghealth-and-safety-during-Covid-19v2.pdf

Center for disease Control and Prevention. (2020, febrero 11). Considerations for Wearing Cloth Face Coverings. Centers for Disease Control and Prevention. https://www.cdc.gov/coronavirus/2019-ncov/prevent-getting-sick/cloth-facecover-guidance.html

Cho, R., Yeung, Z., Ho, O., Lo, J., Siu, A., Kwan, W. M. Y., To, Z. W. H., Chan, A. W. H., Chan, B. Y. T., Fung, K. S. C., Abdullah, V., Tong, M. C. F., \& Ku, P. K. M. (2020). Pearls of experience for safe and efficient hospital practices in otorhinolaryngology - Head and neck surgery in Hong Kong during the 2019 novel coronavirus disease (COVID-19) pandemic. Journal of Otolaryngology Head \& Neck Surgery, 49(1), 1-9. https://doi.org/10.1186/s40463-020-00427-4

Envoiromental Protection Agency. (2020, marzo 13). List N: Disinfectants for Use Against SARS-CoV-2 (COVID-19) [Overviews and Factsheets]. US EPA. https://www.epa.gov/pesticide-registration/list-n-disinfectants-coronaviruscovid-19

Goldin, A., Weinstein, B., \& Shiman, N. (2020). How Do Medical Masks Degrade Speech Reception? - Hearing Review. The Hearing Review. https://www.hearingreview.com/hearing-loss/health-wellness/how-do-medicalmasks-degrade-speech-reception

Hernández-González, S., Monroy, J. F., Rodríguez-Ruiz, M. T., Gutiérrez-Gómez, E., Villegas-Echeverri, M. C., Guerrero, D., \& Quintero, A. (2020). Recomendaciones ante el COVID-19 para la práctica de otología y audiología. Acta De Otorrinolaringología \& Cirugía De Cabeza Y Cuello, 48(1), 55-63. https://doi.org/10.37076/acorl.v48i1.484 
Krajewska, J., Krajewski, W., Zub, K., \& Zatoński, T. (2020). Review of practical recommendations for otolaryngologists and head and neck surgeons during the COVID-19 pandemic. Auris, Nasus, Larynx, 47(4), 544-558. https://doi.org/10.1016/j.anl.2020.05.022

Lavilla, M. J., Huarte, A., Cavallé, L., Núñez, F., \& Juan, E. (2020). Medidas de desinfección e higiene en audiología y estrategias comunicativas durante pandemia COVID-19. Sociedad Española de Otorrinolaringología. Recuperado el 02 Julio de $2020 \mathrm{https}$ ://seorl.net/wp-content/uploads/2020/04/MEDIDAS-DEDESINFECCIÓN-E-HIGIENE-EN-AUDIOLOGÍA-Y-ESTRATEGIASCOMUNICATIVAS-DURANTE-LA-PANDEMIA-COVID-19-1.pdf

Massachusetts Commission for the Deaf and Hard of Hearing. (2020). Covid-19 Card. https://www.mass.gov/doc/covid-19-card/download

Ministerio de Salud. (2020). Casos confirmados en Chile COVID-19. Ministerio de Salud - Gobierno de Chile. https://www.minsal.cl/nuevo-coronavirus-2019ncov/casos-confirmados-en-chile-covid-19/

Moats, S., \& Creel, L. (2020, junio 3). Newborn Hearing Screening and FollowUp. Essential During a Pandemic? Audiology. https://www.audiology.org/audiology-today-mayjune-2020/online-featurenewborn-hearing-screenings-and-follow-essential-during

National Hearing Conservation Program. (2020). Best Practices Guidelines for Safely Conducting Hearing Testing During the COVID-19 Pandemic. https://nhc.memberclicks.net/assets/NHCA\%20COVID-

19\%20Guidelines\%20v1.0.pdf
Organización Mundial de la Salud. (2009). Manual técnico de referencia para la higiene de las manos. https://apps.who.int/iris/bitstream/handle/10665/102537/WHO_IER_PSP_2009. 02_spa.pdf;jsessionid $=3008$ FA8B3DCB74251C84BC8EA43DA89A sequence $=$ 1

Organización Mundial de la Salud. (2020). WHO Coronavirus Disease (COVID19) Dashboard. https://covid19.who.int

Pattisapu, P., Evans, S., Noble, A., Norton, S., Ou, H., Sie, K., \& Horn, D. (2020). Defining Essential Services for Deaf and Hard of Hearing Children during the COVID-19 Pandemic. Otolaryngology-Head and Neck Surgery, 163(1), 91-93. https://doi.org/10.1177/0194599820925058

Rizk, H., Strange, C., Atallah, S., Massingale, S., \& Clendaniel, R. (2020). Coronavirus Disease 2019 Return to Work Guidance and Recommendations for Vestibular Clinicians. Ear and Hearing, 41(4), 693-696. https://doi.org/10.1097/AUD.0000000000000903

Rothan, H. A., \& Byrareddy, S. N. (2020). The epidemiology and pathogenesis of coronavirus disease (COVID-19) outbreak. Journal of Autoimmunity, 109, 1-4. https://doi.org/10.1016/j.jaut.2020.102433

Shi, Y., Wang, G., Cai, X., Deng, J., Zheng, L., Zhu, H., Zheng, M., Yang, B., \& Chen, Z. (2020). An overview of COVID-19. Journal of Zhejiang UniversityScience B, 21(5), 343-360. https://doi.org/10.1631/jzus.B2000083

West, J., Franck, K., \& Welling, D. (2020). Providing health care to patients with hearing loss during COVID-19 and physical distancing. Laryngoscope Investigative Otolaryngology, 5(3), 396-398. https://doi.org/10.1002/lio2.382 